\title{
Evaluation of the synergistic olfactory effects of diacetyl, acetaldehyde, and acetoin in a yogurt matrix using odor threshold, aroma intensity, and electronic nose analyses
}

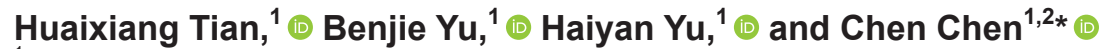 \\ ${ }^{1}$ Department of Food Science and Technology, Shanghai Institute of Technology, Shanghai 201418, China \\ ${ }^{2}$ Shanghai Research Institute of Fragrance and Flavor Industry, Shanghai 200232, China
}

\section{ABSTRACT}

Despite intensive analyses of yogurt flavor, the synergistic effects of the key aroma compounds on sensory responses and their optimum concentration ranges remain less well-documented. This study investigated the odor thresholds, optimum concentration ranges, and perceptual actions of diacetyl, acetaldehyde, and acetoin in a yogurt matrix. Our results show that the odor thresholds of diacetyl, acetaldehyde, and acetoin in the yogurt matrix were $5.43,15.4$, and $29.0 \mathrm{mg} / \mathrm{L}$, respectively, which were significantly higher than the corresponding values in water. The optimum diacetyl, acetaldehyde, and acetoin concentration ranges were found to be 6.65 to $9.12,25.9$ to 35.5 , and 37.3 to $49.9 \mathrm{mg} / \mathrm{L}$, respectively. In Feller's additive model, the addition of each compound led to a significant reduction in their odor threshold in the yogurt matrix, thus demonstrating the synergistic effects of the compounds. In the $\sigma-\tau$ plot, various concentrations of compounds were associated with various degrees of additive behavior with respect to the aroma intensity of the yogurt matrix, thus demonstrating the synergism among these compounds in increasing the overall aroma intensity. The optimal simultaneous concentration ratio of diacetyl:acetaldehyde:acetoin was determined to be 4.00:16.0:32.0 mg/L. The specific synergistic effects were also confirmed by an electronic nose analysis and aroma profile comparison. In summary, these 3 aroma compounds exhibited synergistic effects in a yogurt matrix, thus providing a theoretical basis for the enhancement of flavors in dairy products.

Key words: yogurt, key aroma compound, threshold, synergism

Received August 26, 2019.

Accepted May 5, 2020.

*Corresponding author: chenchen@sit.edu.cn

\section{INTRODUCTION}

Yogurt is a dairy product produced by the fermentation of milk by lactic acid bacteria (LAB). It often has a smooth, viscous gel-like texture and a characteristic sharply acidic, green apple-like flavor (Casarotti et al., 2014; Chen et al., 2017). This distinct flavor is produced by lactic acid and a complex mixture of aroma compounds, which mainly include volatile compounds already present in milk and specific compounds produced during fermentation (Cheng, 2010). To date, more than 90 volatile compounds have been identified in yogurt, including carbohydrates, alcohols, aldehydes, ketones, acids, esters, lactones, sulfur-containing compounds, pyrazines, and furan derivatives (Cheng, 2010; Güler and Gürsoy-Balc1, 2011).

However, not all of these identified volatile compounds are key aroma compounds, which usually refer to characteristic compounds that are indispensable for imparting a desirable flavor to yogurt (Patel et al., 2013; Mercedes et al., 2020). In particular, numerous studies have focused on identifying key odorants in yogurt products via aroma recombination and omission analyses (McGorrin, 2007; Wang et al., 2020). Although no consistent conclusions have been drawn regarding the key aroma compounds in yogurt, some compounds such as diacetyl, acetaldehyde, and acetoin have been widely recognized to add desirable flavors to yogurt (Rysstad and Abrahamsen,1987; Rankin and Bodyfelt, 1995). Of these compounds, acetaldehyde imparts a fresh, green flavor and is considered to be the most important contributor to the typical yogurt aroma (Lockey et al., 2009). Diacetyl imparts a sweet, buttery, creamy, and milky flavor and is commonly used to enhance buttery flavors, and acetoin reduces the stimulating aroma of high-concentration diacetyl and imparts a mild creamy flavor (West, 2020). These compounds are often used to improve the flavor of yogurt either via production by $\mathrm{LAB}$ fermentation or via direct addition as food flavor enhancers (Ping et al., 2018). 
As mentioned above, the odor threshold is an important indicator of a compound's contribution to flavor. Existing data on the odor thresholds of diacetyl, acetaldehyde, and acetoin $(0.003,0.11$, and $8 \mathrm{mg} / \mathrm{L}$, respectively) were mostly obtained via analyses of aqueous solutions, such as a water matrix (Cheng, 2010). Importantly, the actual threshold values of these compounds vary depending on the matrix they occupy (van Aardt et al., 2001). Food macronutrients, such as fat, protein, and carbohydrates, can significantly affect the release of volatile compounds (Haahr et al., 2000; Mende et al., 2016). For example, Adhikari et al. (2006) found that the odor thresholds of diacetyl and other compounds differ significantly between water and skim milk matrices. Although diacetyl, acetaldehyde, and acetoin are considered the most potent flavor compounds in yogurt to our knowledge, the odor thresholds of these compounds in a yogurt matrix have not been reported (Adhikari et al., 2006).

A series of studies on flavor improvement and sensory acceptance have shown that suitable concentrations of aroma compounds may impart a desirable flavor to a food, whereas excess amounts may lead to off flavors and reduced acceptance by consumers (Kang et al., 2018; Vanegas-Azuero and Gutiérrez, 2018). For example, a pungent and irritating odor is associated with high concentrations of acetaldehyde, and a high diacetyl concentration ( $>10 \mathrm{mg} / \mathrm{L}$ ) imparts an undesirable buttery flavor to beer (Cox-Ganser et al., 2011; Afzal et al., 2017). Accordingly, a desirable flavor in yogurt would require that the major flavor compounds are present at appropriate concentrations such that off flavors are not generated.

Researchers have also observed complex perceptual effects of large numbers of aroma compounds on sensory responses, even of those present at concentrations below their individual odor thresholds. Zhu et al. (2017) observed that mixtures of compounds with similar structures and aromas exhibit synergistic effects and additive actions in oolong tea, which enhance the overall aroma intensity. In one study, supplementation with a mixture of R- and S-ethyl 2-hydroxy-4-methylpentanoate in red wines at concentrations 4.5 times lower than their average concentrations led to the perception of a fruity characteristic (Lytra et al., 2012). Similarly, some synergistic effects of aroma compounds in yogurt have also been identified (Gardini et al., 1999; Chaves et al., 2002). For example, excess acetaldehyde relative to diacetyl is reported to yield a green off flavor (Imhof et al., 1995). In addition, the flavor of acetoin tends to reduce the harshness of diacetyl-imparted flavor (Cheng, 2010). A previous report suggested that an acetaldehyde-to-acetone ratio of 2.8:1 can impart a desired fullness and flavor to yogurt (Cheng, 2010). However, previous studies have not provided adequate information about the interrelationships and ideal ratios of these key aroma compounds in yogurt.

In this study, we first evaluated the odor thresholds and then determined the suitable concentration ranges of diacetyl, acetaldehyde, and acetoin in a yogurt matrix. Finally, we evaluated the synergistic effects of these 3 compounds using Feller's additive model and a $\sigma-\tau$ plot and verified them by electronic nose analysis. The results revealed various degrees of synergism among the 3 aroma compounds, depending on their thresholds and concentrations. This newly identified phenomenon of synergism provides additional theoretical support for the knowledge of key aroma compounds in yogurt and the potential for improvements in the organoleptic qualities of the final products.

\section{MATERIALS AND METHODS}

\section{Materials and Chemicals}

Skim milk powder (Fonterra, Auckland, New Zealand) was used to reconstitute milk in a yogurt matrix preparation. The diacetyl, acetaldehyde, and acetoin (all chromatographic grade, $\geq 97 \%$ purity) used in the sensory tests were purchased from Sigma-Aldrich (Shanghai, China), and the lactic acid solution (food grade, $\geq 80 \% \mathrm{vol} / \mathrm{vol}$ ) used for $\mathrm{pH}$ adjustment of the yogurt matrix was purchased from Jindan Co. Ltd. (Henan, China).

\section{Matrix Preparation}

The yogurt matrix was prepared and the aroma compounds were added as described by van Aardt et al. (2001) with some modifications. Reconstituted milk $(12 \% \mathrm{wt} / \mathrm{vol})$ was prepared from skim milk powder before matrix preparation. Modified starch $(1 \% \mathrm{~m} / \mathrm{v})$ and pectin $(0.1 \% \mathrm{~m} / \mathrm{v})$ were then added to the reconstituted milk and mixed with a blender $(2,000 \mathrm{rpm} / \mathrm{min}$, $55^{\circ} \mathrm{C}$ for $\left.30 \mathrm{~min}\right)$ to obtain a yogurt matrix with the appropriate texture. The final composition of the yogurt matrix was $0.170 \%$ fat, $2.34 \%$ protein, and $6.75 \%$ nonfat milk solids. The mixture was then homogenized (2 stages, $15.00 / 5.00 \mathrm{MPa}, 60^{\circ} \mathrm{C}$ ) using a homogenizer (FB-110Q, Litu, Shanghai, China) and pasteurized at $95^{\circ} \mathrm{C}$ for $5 \mathrm{~min}$. The $\mathrm{pH}$ of the yogurt matrix was adjusted to 4.6 with a lactic acid solution. Next, the aroma compounds were added to the yogurt matrix at the required concentrations. The matrix was mixed gently using a blender $(1,000 \mathrm{rpm} / \mathrm{min}$ for $2 \mathrm{~min})$ and refrigerated at $4^{\circ} \mathrm{C}$ until testing. 


\section{General Conditions for Sensory Tests}

The sensory tests were performed as described in previous reports with some modifications (Drake, 2007; Lytra et al., 2014,2015). The prepared yogurt samples were enclosed in individual brown glass bottles, which were coded with random 3-digit numbers, at controlled room temperature. Each sensory test session had a duration of approximately $5 \mathrm{~min}$.

For the sensory evaluation experiment, we recruited candidates with previous experience in olfactory tests and no declared deficiencies in their senses of smell. These candidates underwent discriminate selections 3 times per week over a period of $2 \mathrm{wk}$ to select panelists who could precisely discriminate various yogurt flavors. The panelists (25 men and 20 women, age: $35 \pm 10$ yr) were selected from 150 candidates, including yogurt researchers. The selected panelists underwent a series of training sessions on the detection, recognition, and identification of yogurt flavors before the experiment began. The specific sensory evaluation experiment was designed with reference to the following experimental contents.

\section{Measurement of Diacetyl, Acetaldehyde, and Acetoin Odor Thresholds in the Yogurt Matrix}

The odor thresholds of diacetyl, acetaldehyde, and acetoin in the yogurt matrix were measured using the ASTM-E1432 method (ATSM International, 2011a). A series of test samples were prepared by dispersing a gradient of compounds into the yogurt matrix with a dilution factor of 2.0. The panelists were presented with ten 3-alternative forced-choice presentation (3-AFC) tests. The initial concentrations of diacetyl, acetaldehyde, and acetoin in the yogurt matrix were 0.0003, 0.11 , and $8 \mathrm{mg} / \mathrm{L}$, respectively, according to their odor thresholds in water. The panelists began the stimulation at the highest concentrations, which were expected to correspond to 2 concentration steps above the estimated thresholds. If a panelist could recognize the aroma of the same sample each time, the sample of the next lower concentration was tested in a similar manner. The threshold was defined as the concentration at which the correct detection probability was $50 \%$. The concentration function is a psychometric function that can be well represented by the sigmoid curve $y=1$ / $\left.\left[1+e^{(-\lambda x}\right)\right]$, where $\lambda$ is a constant parameter in the sigmoid curve. The detection probability was corrected using the chance factor $P=(3 \times p-1) / 2$, where $p$ represents the proportion of correct responses for each concentration and $P$ represents the proportion of responses corrected by the chance effect ( $1 / 3$ for 3 -AFC). All experiments were performed in triplicate.

\section{Determination of the Optimum Concentration Ranges of Diacetyl, Acetaldehyde, and Acetoin in the Yogurt Matrix}

The optimum concentration ranges of diacetyl, acetaldehyde, and acetoin were determined using methods described in previous studies with some modifications (Lawless et al., 2000; Walter and Boakes, 2009; Rababah et al., 2012). The concentration ranges were divided into 9 logarithmically spaced intervals; logarithmic (rather than arithmetic) stimulus spacing was used to conform to the Fechnerian (Semilog) function (Walter and Boakes, 2009). The threshold concentration was set at the third concentration point level, and the concentration ranges are described in detail in Table 1. A series of single-compound samples at various concentrations was presented in a random order. Each panelist estimated the total intensity of the aroma at least 3 times per concentration. The ideal concentration ranges were reported by all of the panelists. The data were summarized as the geometric means of all panelists' ideal concentration ranges with standard deviations.

\section{Perceptual Response Evaluated by Feller's Additive Model}

The synergistic effects of mixtures of diacetyl, acetaldehyde, and acetoin were evaluated using Feller's additive model, as adapted by Toshio et al. (2009). Three pairs of binary mixtures were obtained, and the actual model obtained from the experiment of the mixture was compared with a simple theory model. The theory

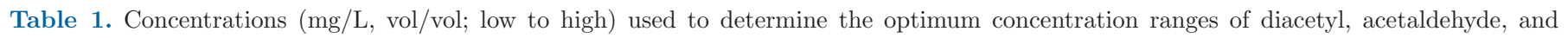
acetoin in a yogurt matrix

\begin{tabular}{|c|c|c|c|c|c|c|c|c|c|}
\hline \multicolumn{4}{|l|}{ Compound } & \multicolumn{6}{|c|}{ Concentration range $^{1}(\mathrm{mg} / \mathrm{L})$} \\
\hline Diacetyl & 2.71 & 3.84 & 5.43 & 7.67 & 10.9 & 15.4 & 21.71 & 30.7 & 43.4 \\
\hline Acetaldehyde & 7.69 & 10.9 & 15.4 & 21.8 & 30.8 & 43.5 & 61.5 & 87.0 & 123 \\
\hline Acetoin & 14.5 & 20.5 & 29.08 & 41.0 & 57.9 & 82.0 & 116 & 164 & 232 \\
\hline
\end{tabular}

${ }^{1}$ The number of significant figures that can be reported is based on the number of digits in the least precise number given. 
probability of each binary mixture was defined as follows: $p(A B)=p(A)+p(B)-p(A) p(B)$, where $p(A)$ and $p(B)$ represent the probability of component $A$ and $B$ detected by the 3 -AFC method, respectively. The measurement probability $p(A B)$ was also determined by the 3-AFC method. The curves for the actual and theoretical models were generated by the data from measured and theoretical $p(A B)$, respectively. Then, the olfactory threshold was defined as the point at which the detection probability was $50 \%$, and the actual and theoretical thresholds for each binary mixture were obtained. If the theoretical threshold for the mixture exceeded the actual value, some degree of synergistic or enhanced effect was considered to have occurred. In contrast, if theoretical threshold was below the actual value, a certain degree of inhibition was considered to have occurred. Finally, if the 2 thresholds were equal, we considered there to be no perceptual effects on sensory responses.

\section{Odor Intensity Testing Using the $\sigma$-т Plot}

Four concentrations (approximately the threshold of each compound) of the 3 compounds were used to prepare the binary mixtures. The selected concentrations were close to the respective thresholds $(2.00,4.00,8.00$, and $16.0 \mathrm{mg} / \mathrm{L}$ for diacetyl; 4.00, 8.00, 16.0, and 32.0 $\mathrm{mg} / \mathrm{L}$ for acetaldehyde; and 8.00, 16.0, 32.0, and 64.0 $\mathrm{mg} / \mathrm{L}$ for acetoin). For each pair of compounds, the odor intensity was detected for 16 binary mixtures $(4 \times$ 4) and 8 single mixtures. Each sample was presented 3 times during each session. For each sample, the panelists rated the odor intensity of the yogurt flavor on a $10-\mathrm{cm}$ scale printed on paper, labeled from 0 on the left, representing no odor perceived, to 10 on the right, representing very intense. The mean experimental data were presented using a synthetic representation based on the 2 parameters of $[\sigma=f(\tau)]$ for binary mixtures, as proposed by Lytra et al. (2013) and Cameleyre et al. (2015). Here, $\tau$ refers to the ratio of the perceived intensity of the binary mixture to the sum of the perceived intensities of the individual components before mixing. The calculation formula is $\tau=I_{A} /\left(I_{A}+I_{B}\right)$, where $I_{A}$ and $I_{B}$ are the perceived odor intensities of components $A$ and $B$, respectively, in the mixture at different concentrations. In addition, $\sigma$ reflects the ratio of the perceived intensity of the mixture and the sum of the perceived intensities of each signal component, and reflects the level of various perceptual intensities. The calculation formula is $\sigma=I_{m i x} /\left(I_{A}+I_{B}\right)$, where $I_{\text {mix }}$ is the perceived odor intensity of the mixture. Both $\tau$ and $\sigma$ were calculated for the aroma intensity.
The positions of experimental points in the graph reflect the levels of perceptual intensities. Cain and Drexler (1974) referred to mixture intensity as the overall perceived intensity of a mixture relative to the sum of the perceived intensities of the unmixed components. They separated several cases of the perceived intensity of a mixture as $\sigma=1, \sigma>1$, and $\sigma<1$, representing complete addition, hyper-addition, and hypo-addition, respectively. Frijters (1987) further differentiated hypoaddition into 3 cases, partial addition, compromise, and subtraction, reflecting whether the intensity of a mixture is greater than, intermediate to, or less than that of the individual compounds, respectively.

\section{Electronic Nose Analysis}

To further verify the synergistic effects of diacetyl, acetaldehyde, and acetoin, 4, 16, and $32 \mathrm{mg} / \mathrm{L}$ were selected as their detected concentrations, respectively. Single, binary, and ternary mixtures of these compounds were generated and subjected to an aroma profile estimation using an electronic nose system (FOX 4000 nose, Alpha MOS, Toulouse, France) coupled with 18 metal oxide sensors (LY2/LG, LY2/G, LY2/AA, LY2/ GH, LY2/gCTL, LY2/gCT, T30/1, P10/1, P10/2, $\mathrm{P} 40 / 1, \mathrm{~T} 70 / 2, \mathrm{PA} / 2, \mathrm{P} 30 / 1, \mathrm{P} 40 / 2, \mathrm{P} 30 / 2, \mathrm{~T} 40 / 2$, $\mathrm{T} 40 / 1$, and $\mathrm{TA} / 2$; Wu et al., 2016).

Briefly, $1.50 \mathrm{~g}$ of each mixture was prepared in a $10-\mathrm{mL}$ glass vial. The vials were placed in order in the headspace system of an automatic sampler. Each vial was incubated at $50^{\circ} \mathrm{C}$ for $300 \mathrm{~s}$ under agitation $(500$ $\mathrm{rpm} / \mathrm{min}$ ), and $2 \mathrm{~L}$ of headspace air was then injected into the E-nose at the rate of $150 \mathrm{~mL} / \mathrm{min}$. Sensor resistance was measured for $120 \mathrm{~s}$ at the rate of 1 acquisition per second. Each sample was analyzed 6 times, and the average of the final 3 analyses was used in the final data set. After each analysis, the values for all samples were adjusted by subtracting the value of the blank yogurt matrix. Finally, the value of each mixture was compared with the sum of each separate component.

\section{Statistical Analysis}

Sigma Plot 12.0 software (SYSTAT Inc., Chicago, IL) was used to fit Feller's additive model, and Origin 9.0 (OriginLab Corporation, Northampton, MA) was used to draw the figures. The statistical analysis of electronic nose data was performed using Alpha-soft version 12.4 software (Alpha MOS, Toulouse, France). All statistical data were analyzed using XLSTAT 7.5 (Addinsoft, Long Island City, NY), and Duncan's test was used to determine the statistical differences. A $P$ - 
value of $<0.05$ was considered to indicate statistical significance.

\section{RESULTS AND DISCUSSION}

\section{Thresholds of Diacetyl, Acetaldehyde, and Acetoin in the Yogurt Matrix}

Table 2 presents the statistical results of the threshold test findings. The detected odor thresholds of diacetyl, acetaldehyde, and acetoin differed significantly from the values reported in the literature $(P<0.05)$. Particularly, the thresholds of diacetyl and acetaldehyde in the yogurt matrix were 1,810- and 139-fold higher, respectively, than the corresponding values detected in water. This difference in threshold values is likely attributable to the different matrices. Previous reports have suggested that the final flavor perception is influenced by any chemical interactions between the food components (Mende et al., 2016). The complex macronutrients in yogurt (fat, protein, and carbohydrates) may mask the perception of volatile compounds (Mende et al., 2016). In addition, the thresholds in our study were detected using the ASTM-E1432 method rather than the ASTM-E679-041 method (ATSM International, 2011a, b). Because the latter does not use the chance factor to correct the detection probability, this may have resulted in the differences in the threshold values. Collectively, the actual thresholds of these 3 compounds in the yogurt matrix were significantly higher than those in water, as reported in the literature, and these differences may have affected their contributions to the overall aroma of yogurt.

\section{Optimum Concentrations of Diacetyl, Acetaldehyde, and Acetoin in the Yogurt Matrix}

According to the literature, both high and low concentrations of aroma compounds can result in off flavors, indicating that an appealing flavor in yogurt can result only when the key aroma compounds are present in the appropriate concentrations (Lee et al., 2007; Cox-Ganser et al., 2011). In this study, we found that low concentrations (i.e., slightly above the threshold) of diacetyl, acetaldehyde, and acetoin often resulted in a weak flavor. In contract, excessively high concentrations resulted in a harsh flavor. Accordingly, we determined the optimum concentration ranges of diacetyl, acetaldehyde, and acetoin to be 6.65 to 9.12 , 25.9 to 35.5 , and 37.3 to $49.9 \mathrm{mg} / \mathrm{L}$, respectively (Table 3 ). The literature suggests that in yogurt, 23 to $40 \mathrm{mg} /$ $\mathrm{kg}$ of acetaldehyde yields a good flavor, which is consistent with our findings (Gaafar, 1992). Furthermore, the typical concentrations of diacetyl and acetoin in yogurt range from 0.2 to 3 and from 1.2 to $28.2 \mathrm{mg} / \mathrm{kg}$, respectively (Pourahmad and Assadi, 2005). However, the optimum concentration ranges of diacetyl and acetoin determined in our study were significantly higher than those values, and these differences are probably due to the different matrices used to determine the optimum concentrations. For example, the use of milk matrix has been reported to have a distinct effect on the optimum concentration range of acetaldehyde compared with the use of a chocolate milk or water matrix (van Aardt et al., 2001).

\section{Synergistic Effects Evaluated Using Feller's Additive Model}

Although the odor threshold value of a compound is a good indicator of its olfactory effect, it is inappropriate to assume that the sum of the contributions made by individual compounds can reflect the overall flavor because this approach neglects several potential synergistic effects that affect odor perception (Cameleyre et al., 2015). Therefore, we used Feller's additive model to evaluate the synergistic effects of these compounds. Experimentally, the results showed that the actual experimental detection probabilities were higher than those fit by the theoretical formula in Feller's addi-

Table 2. Detection thresholds of diacetyl, acetaldehyde, and acetoin in a yogurt matrix

\begin{tabular}{|c|c|c|c|c|}
\hline Compound & Aroma description $^{1}$ & \multicolumn{2}{|l|}{ Threshold } & Threshold, literature $^{2}(\mathrm{mg} / \mathrm{L})$ \\
\hline Diacetyl & Sweet, buttery, creamy, milky & 5.43 & 0.910 & 0.003 \\
\hline Acetoin & Creamy, dairy, sweet, milky, buttery & 29.0 & 0.860 & 8 \\
\hline
\end{tabular}

${ }^{1}$ Description of the aromas of the 3 compounds in yogurt.

${ }^{2}$ Thresholds of the 3 compounds reported in the literature.

${ }^{3}$ Thresholds of the 3 compounds determined in this study.

${ }^{4} \mathrm{SD}$ of the thresholds of the 3 compounds. 
tive model (Figure 1) when each pair was added to the yogurt matrix. The ratios of the actual and theoretical detection thresholds ranged from a minimum factor of 1.81 for the acetaldehyde and acetoin pair (Figure 1c) to 2.31 for the diacetyl and acetoin pair (Figure 1b). This suggested that, due to the similar aromas of diacetyl and acetoin, this pair may yield the strongest synergistic effects. This finding clearly demonstrated the synergistic effects of the 3 pairs of compounds; all 3 were carbonyl compounds, and thus structural similarities may have led to these synergistic effects (Zhu et al., 2017).

\section{Synergistic Effects of the Binary Mixture Detected by the $\sigma-\tau$ Plot}

Although the existence of synergy between the 3 compounds has been proven, the degrees of synergistic effects when these compounds are present in different concentrations remains unknown. Here, we applied the $\sigma-\tau$ plot approach to measure changes in the perceptual intensities of the mixtures along with the concentrations of the components that were altered. Meanwhile, the functional ratios of the 3 compounds were also determined. According to the $\sigma=\mathrm{f}(\tau)$ representation, the experimental results were stratified into 5 categories according to the level of interaction (Niu et al., 2018).

As shown in Figure 2, most points were located in the region of partial addition. In other words, at those concentrations, the perceptual intensity of the mixture was weaker than the sum of the intensities of the individual components, but stronger than the intensities of the single compounds. Of the 72 mixtures, 5 were located in the region of hyper-additivity $(\sigma>1.05)$, whereas only 1 was located in the region of perfect additivity $(0.95$ $<\sigma<1.05)$. One point located in the region of hyperaddition in Figure 3a had $\tau$ values of 0.473 in Figure 2a, panel 1 and 0.541 in Figure 2a, panel 2. The corresponding concentrations were $4.00 \mathrm{mg} / \mathrm{L}$ for diacetyl and $16.0 \mathrm{mg} / \mathrm{L}$ for acetaldehyde. In Figure $2 \mathrm{~b}, 3$ points located in the region of hyper-addition had correspond- ing $\tau$ values of $0.472,0.753$, and 0.313 in Figure $2 b$, panel 1 and 0.716, 0.454, and 0.752 in Figure 2b, panel 2 . The corresponding pairs of diacetyl and acetoin concentrations were 4.00 to $16.0,4.00$ to 32.0 , and 16.0 to $32.0 \mathrm{mg} / \mathrm{L}$, respectively. For the pairs of acetaldehyde and acetoin concentrations (Figure 2c), 1 mixture $(\tau=$ 0.765 in Figure 2c, panel 1 and $\tau=0.334$ in Figure 2c, panel 2) was classified in the region of hyper-additivity, and one mixture $(\tau=0.792$ in Figure 2c, panel 1 and $\tau=0.152$ in Figure 2c, panel 2) was classified in the region of perfect additivity. Notably, hyper-additivity was observed frequently when the concentrations of the aroma compound pairs were at the threshold level. This finding was consistent with the results reported by $\mathrm{Wu}$ et al. (2012) that flavor is enhanced when the odorants are added at threshold levels, which can contribute to the overall odor of the mixture.

Based on the above results, we determined the hyperaddition ratios of 1:4 for the diacetyl and acetaldehyde pair and 1:2 for the acetaldehyde and acetoin pair. These ratios were consistent with the diacetyl-to-acetaldehyde ratio range of $1: 4.40$ to $1: 3.20$ reported by Lindsay et al. (1967) for flavored whole yogurt. However, Cheng (2010) reported that an acetaldehyde-to-diacetyl ratio of 1:1 would yield a typical and preferable yogurt flavor. These discrepancies may be due to the interference of the matrix and the different perceptions of the synergistic effects of aroma compounds. For the diacetyl and acetoin pair, hyper-addition was observed at ratios of 1:4 and 1:8. Given these perceptual responses to synergistic effects, optimal concentration settings of $4.00,16.0$, and $32.0 \mathrm{mg} / \mathrm{L}$ were determined for diacetyl, acetaldehyde, and acetoin, respectively. Comparison of the threshold values and optimum concentrations between the single compounds and the mixture showed that the best sensory perceptions of the mixture did not occur within the optimum concentration ranges of the individual compounds; in fact, in some cases, they occurred at lower concentrations than the thresholds. This result suggested that a synergistic effect enhanced the overall aroma intensity of the yogurt matrix even

Table 3. Optimum concentrations of diacetyl, acetaldehyde, and acetoin in a yogurt matrix

\begin{tabular}{lccccc}
\hline & \multicolumn{3}{c}{ Optimum concentration detected ${ }^{1}(\mathrm{mg} / \mathrm{L})$} & \multirow{2}{*}{ Concentration reported $^{2}\left(\mathrm{mg}^{2} / \mathrm{L}\right)$} \\
\cline { 2 - 4 } Compound & Minimum & $\mathrm{SD}^{3}$ & Maximum & $\mathrm{SD}^{3}$ & $0.2-3$ \\
\hline Diacetyl & 6.65 & 1.43 & 9.12 & 1.83 & $23-40$ \\
Acetaldehyde & 25.9 & 2.84 & 35.5 & 4.84 & $1.2-28.2$ \\
Acetoin & 37.3 & 3.92 & 49.9 & 4.84 & \\
\hline
\end{tabular}

\footnotetext{
${ }^{1}$ Optimum concentrations of the 3 compounds determined in this study.

${ }^{2}$ Concentrations of the 3 compounds in dairy foods reported in the literature.

${ }^{3} \mathrm{SD}$ of the optimum concentrations of the 3 compounds.
} 
A-B
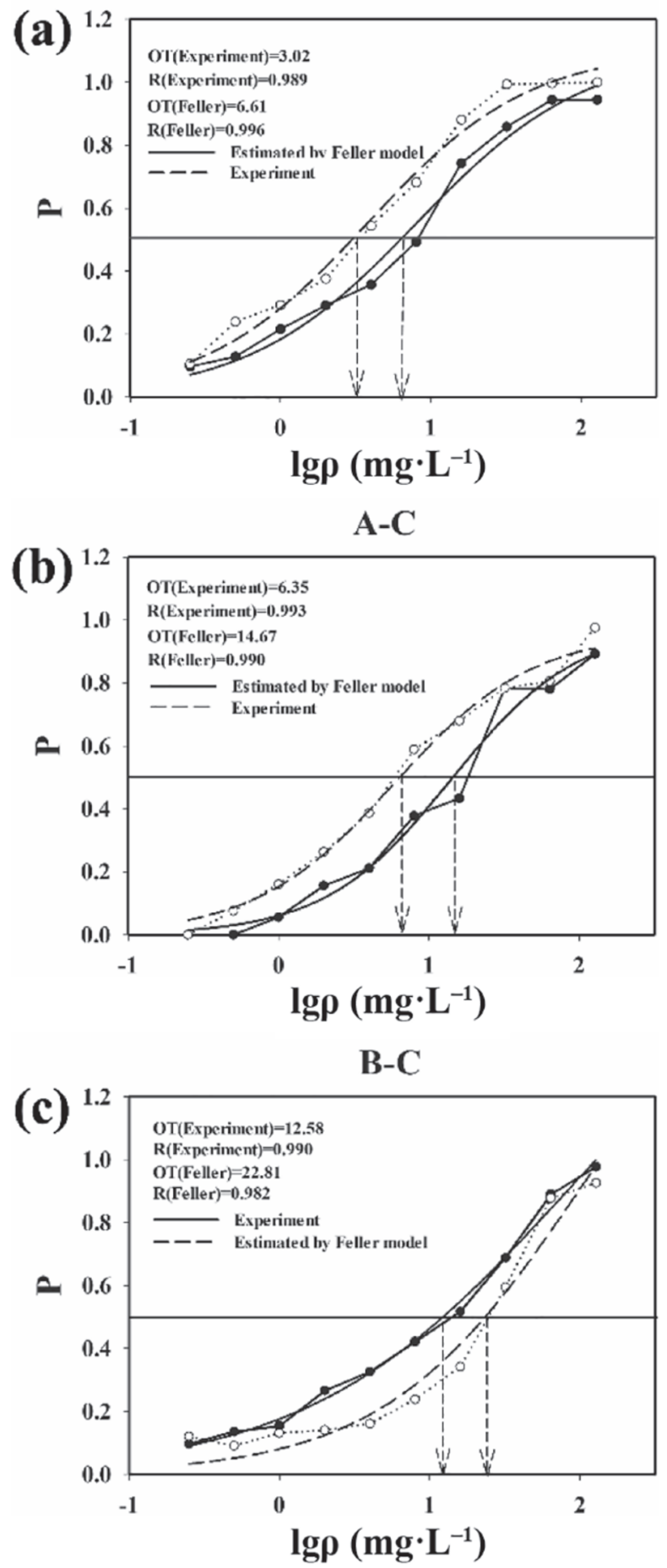

Figure 1. Effect of the addition of diacetyl, acetaldehyde, and acetoin on the detection probability in a yogurt matrix, as determined using Feller's additive model. (a) Diacetyl (A) and acetaldehyde (B) pair, (b) diacetyl (A) and acetoin (C) pair, and (c) acetaldehyde (B) and acetoin $(\mathrm{C})$ pair. $\mathrm{P}=$ proportion of correct responses; $\mathrm{OT}=$ olfactory threshold; $\mathrm{R}=$ Feller's additive model fitting result; $\lg \rho=$ concentration of compounds. In each graph, horizontal line indicates that the correct detection probability is $50 \%$, and the 2 arrows respectively represent the corresponding $\lg \rho$ values under the correct detection probability of $50 \%$. with reduced concentration of a single aroma compound in the mixture.

\section{Synergistic Effects Verified by Electronic Nose Analysis}

The electronic nose can mimic the biological principles of smell and thus provide a holistic view of the profiles of volatile compounds (Wu et al., 2012). To verify the synergistic effects of the 3 aroma compounds on the aroma profile, we subjected the mixtures to electronic nose analysis. As shown in Figure 3, sensors T30/1, P10/1, P10/2, T70/2, PA/2, P30/1, P30/2, $\mathrm{T} 40 / 2$, and $\mathrm{TA} / 2$ reported strong responses, with some obvious differences. As shown in Figure 3b, sensors LY2/LG, P10/1, P10/2, P40/1, T70/2, T40/2, T40/1, and TA $/ 2$ reported significantly stronger responses $(P$ $<0.05)$ to a binary mixture of diacetyl and acetoin compared with the sum of responses to each individual compound. Similar results were also obtained for the acetaldehyde and acetoin pair. The response values of sensors LY2/LG, LY2/G, LY2/AA, LY2/GH, LY2/ gCTL, P10/1, P10/2, P40/1, T40/1, and TA/2 to a binary mixture of diacetyl and acetaldehyde were greater than the sum of responses to each individual compound. When all 3 compounds were mixed together, the characteristic profile shifted considerably, particularly when detected by sensors LY2/G, LY2/ $\mathrm{AA}, \mathrm{P} 10 / 1, \mathrm{P} 10 / 2, \mathrm{P} 40 / 1, \mathrm{~T} 70 / 2, \mathrm{~T} 40 / 2, \mathrm{~T} 40 / 1$, and $\mathrm{TA} / 2$ (Figure $3 \mathrm{~d}$ ). The literature reports that these 9 sensors are sensitive to ketones, aldehydes, or compounds with carbonyl group, indicating that the differences between responses to binary mixtures and individual compounds could be more easily captured by these sensors (Wu et al., 2012). These results indicated changes in the aroma profiles of the mixtures in this study due to the synergistic effects of the aroma compounds, consistent with the results obtained in our previous sensory analysis.

These results confirm the synergistic effects of diacetyl, acetaldehyde, and acetoin in a yogurt matrix, which supported the theory that compounds with similar structures and aromas have synergistic effects on sensory responses (Saison et al., 2009; Niu et al., 2018). This result can also provide a practical guide for yogurt flavor improvement because we can regulate LAB fermentation or the direct addition of these compounds according to their appropriate ratios. Although the mechanism of synergistic effects remains unclear, a recent study that used the peripheral olfactory epithelium of the nose of a mouse reported that odor mixtures can enhance olfactory responses relative to the responses to individual odor compounds, thereby increasing the capacity of the olfactory system to distinguish complex 
(a)
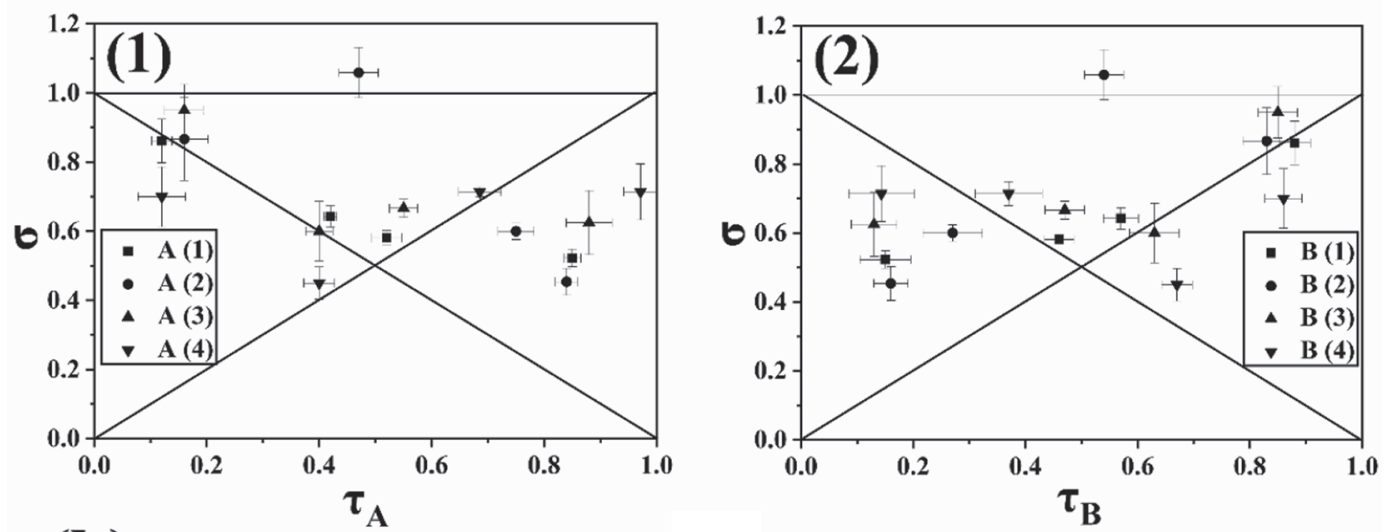

(b)
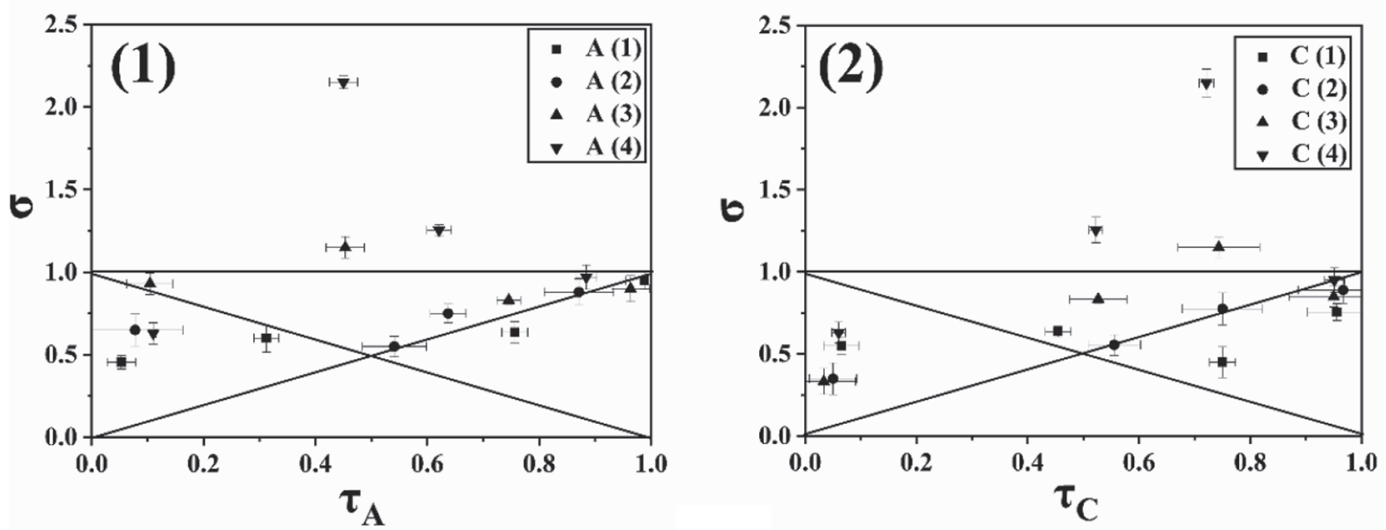

(c)
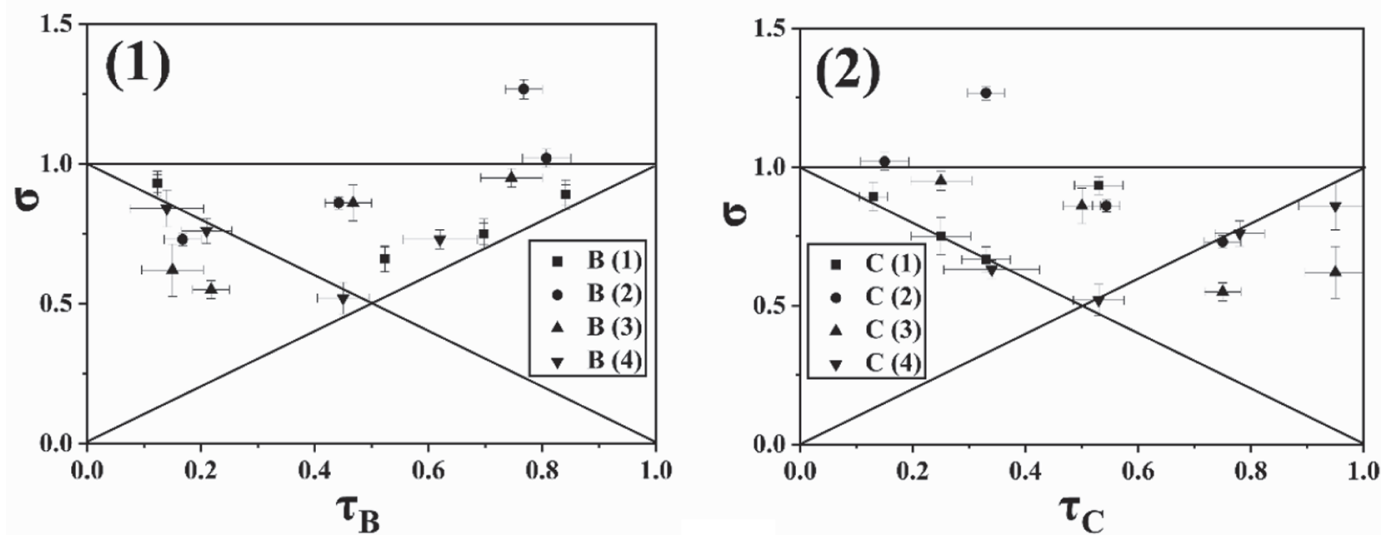

Figure 2. Intensity ratings of the addition of diacetyl, acetaldehyde, and acetoin to the total aroma, as determined using the $\sigma=\mathrm{f}(\tau)$ representation, where $\tau$ refers to the ratio of the perceived intensity of the binary mixture to the sum of the perceived intensities of the individual components before mixing, and $\sigma$ reflects the ratio of the perceived intensity of the mixture and the sum of the perceived intensities of each signal components, reflecting the level of various perceptual intensities. (a) Diacetyl (A) and acetaldehyde (B) pair, (b) diacetyl (A) and acetoin (C) pair, and (c) acetaldehyde (B) and acetoin (C) pair. A (1)-A (4) = 4 concentrations of diacetyl from low to high; B (1)-B (4) = 4 concentrations of acetaldehyde from low to high; C (1)-C (4) = 4 concentrations of acetoin from low to high. Error bars indicate $95 \%$ confidence intervals of the means for both $\tau$ and $\sigma$. 

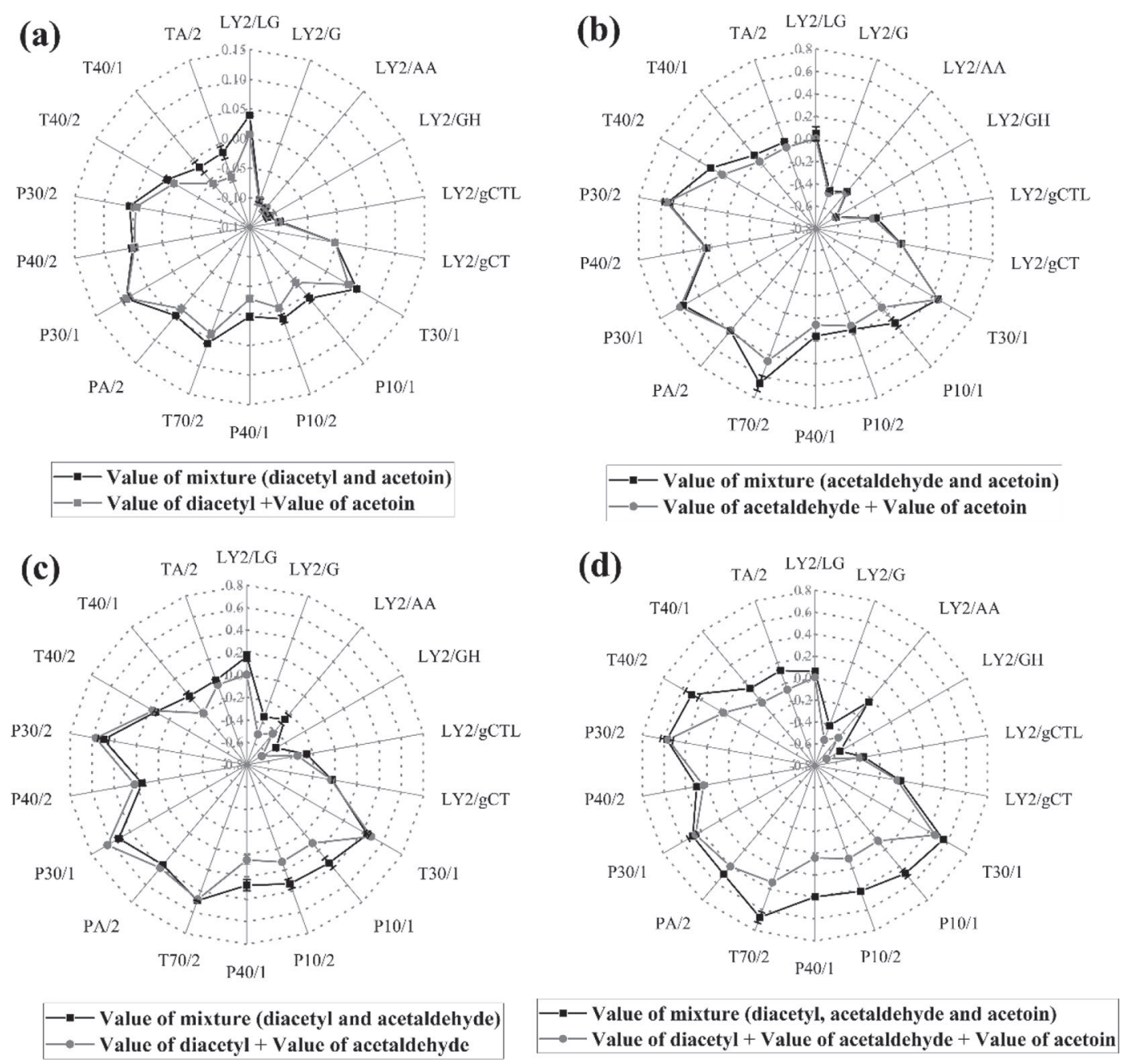

Figure 3. Comparison of aroma profiles according to the value obtained for a mixture versus the sum of the values obtained for each individual compound. (a) Diacetyl and acetoin pair, (b) acetaldehyde and acetoin pair, (c) diacetyl and acetaldehyde pair, and (d) diacetyl, acetaldehyde, and acetoin mixture.

odor mixtures (Xu et al., 2020). The specific underlying mechanism still requires further investigation.

\section{CONCLUSIONS}

In this study, the odor thresholds and optimum concentration ranges of diacetyl, acetaldehyde, and acetoin were determined in a yogurt matrix. In addition, the synergistic effects of these 3 compounds on sensory responses were evaluated using Feller's additive model and the $\sigma-\tau$ plot method and verified with electronic nose analysis. The optimal concentration ratio of diacetyl:acetaldehyde:acetoin was determined to be 4.00:16.0:32.0 mg/L. All of the findings emphasize the importance of the concentrations, synergistic effects, and ratios of the key aroma compounds in yo- gurt. These findings improve our understanding of the volatile compounds responsible for the typical aromas of yogurt, which is expected to help achieve desirable organoleptic properties in the final products by LAB fermentation or direct flavor additions.

\section{ACKNOWLEDGMENTS}

This research was funded by the National Natural Science Foundation of China (Beijing; No. 31972197). This work was also sponsored by the "Shu Guang" project (No. 16SG50), which was supported by the Shanghai Municipal Education Commission, Shanghai Education Development Foundation, and Shanghai Rising-Star Program (No. 17QB1404200; Shanghai, China). The authors have not stated any conflicts of interest. 


\section{REFERENCES}

Adhikari, K., K. A. Hein, J. R. Elmore, H. Heymann, and A. M. Willott. 2006. Flavor threshold as affected by interactions among three dairy-related flavor compounds. J. Sens. Stud. 21:626-643. https://doi.org/10.1111/j.1745-459X.2006.00087.x.

Afzal, M. I., C. C. Ariceaga, K. A. Boulahya, M. Jacquot, S. Delaunay, and C. Cailliez-Grimal. 2017. Biosynthesis and role of 3-methylbutanal in cheese by lactic acid bacteria: Major metabolic pathways, enzymes involved, and strategies for control. Crit. Rev. Food Sci. Nutr. 57:399-406. https://doi.org/10.1080/10408398.2014.893502.

ATSM International. 2011a. Standard practice for defining and calculating individual and group sensory thresholds from forced-choice data sets of intermediate size. ATSM standard E1432-04. ATSM International, West Conshohocken, PA.

ATSM International. 2011b. Standard practice for determination of odor and taste thresholds by a forced-choice ascending concentration series method of limits. ATSM standard E679-7. ATSM International, West Conshohocken, PA.

Cain, W. S., and M. Drexler. 1974. Scope and evaluation of odor counteraction and masking. Ann. N. Y. Acad. Sci. 237:427-439. https: //doi.org/10.1111/j.1749-6632.1974.tb49876.x.

Cameleyre, M., G. Lytra, S. Tempere, and J. C. Barbe. 2015. Olfactory impact of higher 381 alcohols on red wine fruity ester aroma expression in model solution. J. Agric. Food Chem. 63:9777-9788. https://doi.org/10.1021/acs.jafc.5b03489.

Casarotti, S. N., D. A. Monteiro, M. M. S. Moretti, and A. L. B. Penna. 2014. Influence of the combination of probiotic cultures during fermentation and storage of fermented milk. Food Res. Int. 59:67-75. https://doi.org/10.1016/j.foodres.2014.01.068.

Chaves, A. C. S. D., M. Fernandez, A. L. S. Lerayer, I. Mierau, M. Kleerebezem, and J. Hugenholtz. 2002. Metabolic engineering of acetaldehyde production by Streptococcus thermophilus. Appl. Environ. Microbiol. 68:5656-5662. https://doi.org/10.1128/AEM.68 $.11 .5656-5662.2002$.

Chen, C., S. Zhao, G. Hao, H. Yu, H. Tian, and G. Zhao. 2017. Role of lactic acid bacteria on the yogurt flavour: A review. Int. J. Food Prop. 20(Suppl.1):S316-S330. https://doi.org/10.1080/10942912 .2017 .1295988

Cheng, H. 2010. Volatile flavor compounds in yogurt: A review. Crit. Rev. Food Sci. Nutr. 50:938-950. https://doi.org/10.1080/ 10408390903044081.

Cox-Ganser, J., G. Ganser, R. Saito, G. Hobbs, R. Boylstein, W. Hendricks, M. Simmons, M. Eide, G. Kullman, and C. Piacitelli. 2011. Correcting diacetyl concentrations from air samples collected with NIOSH method 2557. J. Occup. Environ. Hyg. 8:59-70. https:// doi.org/10.1080/15459624.2011.540168.

Drake, M. A. 2007. Invited review: Sensory analysis of dairy foods. J. Dairy Sci. 90:4925-4937. https://doi.org/10.3168/jds.2007-0332.

Frijters, J. E. 1987. Psychophysical models for mixtures of tastants and mixtures of odorants. Ann. N. Y. Acad. Sci. 510:67-78. https: //doi.org/10.1111/j.1749-6632.1987.tb43469.x.

Gaafar, A. M. 1992. Volatile flavour compounds of yoghurt. Int. J. Food Sci. Technol. 27:87-91. https://doi.org/10.1111/j.1365-2621 .1992.tb01183.x.

Gardini, F., R. Lanciotti, M. E. Guerzoni, and S. Torriani. 1999. Evaluation of aroma production and survival of Streptococcus thermophilus, Lactobacillus delbrueckii ssp. bulgaricus and Lactobacillus acidophilus in fermented milks. Int. Dairy J. 9:125-134. https: //doi.org/10.1016/S0958-6946(99)00033-3.

Güler, Z., and A. C. Gürsoy-Balcı. 2011. Evaluation of volatile compounds and free fatty acids in set types yogurts made of ewes', goats' milk and their mixture using two different commercial starter cultures during refrigerated storage. Food Chem. 127:10651071. https://doi.org/10.1016/j.foodchem.2011.01.090.

Haahr, A. M., W. L. P. Bredie, and L. H. Stahnke. 2000. Flavour release of aldehydes and diacetyl in oil/water systems. Food Chem. 71:355-362. https://doi.org/10.1016/S0308-8146(00)00184-9.

Imhof, R., H. Glättli, and J. O. Bosset. 1995. Volatile organic compounds produced by thermophilic and mesophilic single strain dairy starter cultures. Lebensm. Wiss. Technol. 28:78-86. https:// doi.org/10.1016/S0023-6438(95)80016-6.

Kang, S.-H., M.-S. Yu, J.-M. Kim, S.-K. Park, and S.-K. Kim. 2018. Biochemical, microbiological, and sensory characteristics of stirred yogurt containing red or green pepper (capsicum annuum cv. chungyang) juice. Korean J Food Sci Anim. Resour. 38:451-467. https: //doi.org/10.5851/kosfa.2018.38.3.451.

Lawless, H. T., J. Horne, and W. Spiers. 2000. Contrast and range effects for category, magnitude and labeled magnitude scales in judgements of sweetness intensity. Chem. Senses 25:85-92. https:/ /doi.org/10.1093/chemse/25.1.85.

Lee, S. H., M. J. Seo, M. Riu, J. P. Cotta, D. E. Block, N. K. Dokoozlian, and S. E. Ebeler. 2007. Vine microclimate and norisoprenoid concentration in Cabernet Sauvignon grapes and wines. Am J. Enol Viticult. 58(3):291-301.

Lindsay, R. C., E. A. Day, and L. A. Sather. 1967. Preparation and evaluation of butter culture flavor concentrates. J. Dairy Sci. 50:25-31. https://doi.org/10.3168/jds.S0022-0302(67)87346-6.

Lockey, J. E., L. P. Hilbert, T. J. Levin, P. H. Ryan, K. L. White, E. K. Borton, C. H. Rice, R. T. McKay, and G. K. LeMasters. 2009 Airway obstruction related to diacetyl exposure at microwave popcorn production facilities. Eur. Respir. J. 34:63-71. https://doi .org/10.1183/09031936.00050808.

Lytra, G., M. Cameleyre, S. Tempere, and J. C. Barbe. 2015. Distribution and organoleptic impact of ethyl 3-hydroxybutanoate enantiomers in wine. J. Agric. Food Chem. 63:10484-10491. https://doi org/10.1021/acs.jafc.5b04332.

Lytra, G., S. Tempere, G. de Revel, and J.-C. Barbe . 2012. Distribution and organoleptic impact of ethyl 2-hydroxy-4-methylpentanoate enantiomers in wine. J. Agric. Food Chem. 60:1503-1509. https://doi.org/10.1021/jf204378u.

Lytra, G., S. Tempere, G. de Revel, and J.-C. Barbe. . 2014. Distribution and organoleptic impact of ethyl 2-methylbutanoate enantiomers in wine. J. Agric. Food Chem. 62:5005-5010. https://doi.org/ 10.1021/jf500670z.

Lytra, G., S. Tempere, A. Le Floch, G. de Revel, and J.-C. Barbe 2013. Study of sensory interactions among red wine fruity esters in a model solution. J. Agric. Food Chem. 61:8504-8513. https://doi .org/10.1021/jf4018405.

McGorrin, R. 2007. Flavor analysis of dairy products. ACS Symp. Ser. 971:23-49. https://doi.org/10.1021/bk-2007-0971.ch002.

Mende, S., H. Rohm, and D. Jaros. 2016. Influence of exopolysaccharides on the structure, texture, stability and sensory properties of yoghurt and related products. Int. Dairy J. 52:57-71. https://doi .org/10.1016/j.idairyj.2015.08.002.

Mercedes, J., P. Majesús, and J. C. Rivas. 2020. Liquid chromatographic determination of carminic acid in yogurt. J. Assoc. Off Anal. Chem. 2:231-234. https://doi.org/10.1093/jaoac/72.2.231.

Miyazawa, T., M. Gallagher, G. Preti, and P. M. Wise . 2009. Odor detection of mixtures of homologous carboxylic acids and coffee aroma compounds by humans. J. Agric. Food Chem. 57:9895-9901. https://doi.org/10.1021/jf901453r.

Niu, Y., Z. Yao, Z. Xiao, G. Zhu, J. Zhu, and J. Chen. 2018. Sensory evaluation of the synergism among ester odorants in light aromatype liquor by odor threshold, aroma intensity and flash GC electronic nose. Food Res. Int. 113:102-114. https://doi.org/10.1016/ j.foodres.2018.01.018.

Patel, A., N. Shah, and J. B. Prajapati. 2013. Biosynthesis of vitamins and enzymes in fermented foods by lactic acid bacteria and related genera - A promising approach. Chem. Biol. Interact. 141:77-95. https://doi.org/10.1016/S0009-2797(02)00067-4.

Ping, L, G Yingying, and W Cailing. 2018. Effect of ILV6 deletion and expression of aldB from Lactobacillus plantarum in Saccharomyces uvarum on diacetyl production and wine flavor. J. Agric. Food Chem. 66:8556-8565. https://doi.org/10.1021/acs.jafc.8b02356

Pourahmad, R., and M. M. Assadi. 2005. Yoghurt production by Iranian native starter cultures. Food Sci. Nutr. 35:410-415. https:// doi.org/10.1108/00346650510633819.

Rababah, T. M., M. A. Al-Mahasneh, W. Yang, R. Esoh, M. N. Alhamad, and M. Al-U'Datt. 2012. Optimizing the best concentration 
of additive flavors to corn chips by evaluating the physicochemical and sensory properties. J. Food Process. Preserv. 36:225-231. https://doi.org/10.1111/j.1745-4549.2011.00582.x.

Rankin, S. A., and F. W. Bodyfelt. 1995. Solvent desorption dynamic headspace method for diacetyl and acetoin in buttermilk. J. Food Sci. 60:1205-1207. https://doi.org/10.1111/j.1365-2621.1995 .tb04556.x.

Rysstad, G., and R. K. Abrahamsen. 1987. Formation of volatile aroma compounds and carbon dioxide in yogurt starter grown in cows' and goats' milk. J. Dairy Res. 54:257-266. https://doi.org/ 10.1017/S0022029900025395.

Saison, D., D. P. De Schutter, B. Uyttenhove, F. Delvaux, and F. R. Delvaux. 2009. Contribution of staling compounds to the aged flavour of lager beer by studying their flavour thresholds. Food Chem. 114:1206-1215. https://doi.org/10.1016/j.foodchem.2008 .10.078.

van Aardt, M., S. E. Duncan, D. Bourne, J. E. Marcy, T. E. Long, C. R. Hackney, and C. Heisey. 2001. Flavor threshold for acetaldehyde in milk, chocolate milk, and spring water using solid phase microextraction gas chromatography for quantification. J. Agric. Food Chem. 49:1377-1381. https://doi.org/10.1021/jf001069t.

Vanegas-Azuero, A. M., and L. F. Gutiérrez. 2018. Physicochemical and sensory properties of yogurts containing sacha inchi (Plukenetia volubilis L.) seeds and $\beta$-glucans from Ganoderma lucidum. J. Dairy Sci. 101:1020-1033. https://doi.org/10.3168/jds.2017-13235.

Walter, F., and R. A. Boakes. 2009. Long-term range effects in hedonic ratings. Food Qual. Prefer. 20:440-449. https://doi.org/10.1016/j .foodqual.2009.03.009.

Wang, B., J. Wang, L. Y. Xu, J. H. Zhang, N. S. Ai, and Y. P. Cao. 2020. Characterization of the key odorants in kurut with aroma recombination and omission studies. Int. Dairy J. https://doi.org/ 10.3168 /jds.2019-17521.

West, D. B. 2020. Determination of diacetyl in beer: Collaborative studies. J. Assoc. Off. Anal. Chem. 61:100-103. https://doi.org/10 $.1093 / \mathrm{jaoac} / 61.1 .100$.

Wu, K. N., B. K. Tan, J. D. Howard, D. B. Conley, and J. A. Gottfried. 2012. Olfactory input is critical for sustaining odor quality codes in human orbitofrontal cortex. Nat. Neurosci. 15:1313-1319 https://doi.org/10.1038/nn.3186

Wu, N., X. C. Wang, N. P. Tao, and Y. Q. Ni. 2016. Odor profiles of hepatopancreas and gonad of Eriocheir sinensis by sensory analysis, electronic nose, and GC-MS analysis. Fish. Sci. 82:537-547. https://doi.org/10.1007/s12562-016-0979-7.

Xu, L, W Li, V Voleti, and D Zou.. 2020. Widespread receptor-driven modulation in peripheral olfactory coding. Sci. 368:eaaz5390. https://doi.org/10.1126/science.aaz5390

Zhu, J., F. Chen, L. Y. Wang, Y. W. Niu, and Z. B. Xiao. 2017. Evaluation of the synergism among volatile compounds in oolong tea infusion by odour threshold with sensory analysis and E-nose. Food Chem. 221:1484-1490. https://doi.org/10.1016/j.foodchem .2016.11.002.

\section{ORCIDS}

Huaixiang Tian @ \ttps://orcid.org/0000-0002-6097-809X

Benjie Yu • https://orcid.org/0000-0003-1564-1137

Haiyan Yu @ https://orcid.org/0000-0001-6472-1366

Chen Chen ๑ https://orcid.org/0000-0002-4885-393X 